\title{
3b-hydroxy-5a-androst-1-en-17-one Increases Testosterone Bioavailability Through Downregulation of Sex Hormone Binding Globulin Protein
}

\author{
Lisa Jansen ${ }^{1}$, Jorge Granados ${ }^{1}$, Halie Harton ${ }^{1}$, Trevor Gillum², Kevin Christmas ${ }^{3}$, and \\ Matthew Kuennen ${ }^{1}$
}

${ }^{1}$ Human Performance Research Laboratory; Department of Sports \& Exercise Sciences; West Texas A\&M University, Canyon, TX

2 Department of Kinesiology; California Baptist University, Riverside, CA

${ }^{3}$ Department of Kinesiology \& Health Education; The University of Texas at Austin; Austin, TX

\section{Category: Masters}

Advisor / Mentor: Kuennen, Matthew (mkuennen@wtamu.edu)

\section{ABSTRACT}

Introduction: In 2012 we reported that the oral prohormone 3b-hydroxy-5a-androst-1-en-17-one improved muscular strength by $13 \%$ and body composition (by augmenting lean body mass $(4.7 \pm 0.8 \mathrm{~kg}$ ), and decreasing fat mass $(2.7 \pm 1.0 \mathrm{~kg})$ ). These changes exceeded those shown in subjects supplemented with an oral placebo who improved muscular strength by $6 \%,(\mathrm{p}=0.002)$, and body composition (by increasing lean body mass $(0.3 \pm 0.5 \mathrm{~kg}),(\mathrm{p}=0.004)$, and decreasing fat mass $(1.2 \pm 0.5 \mathrm{~kg}),(\mathrm{p}=0.014))$. Purpose: The present study examines the mechanism behind these anabolic effects. Methods: 17 resistance-trained males (Age: $23 \pm 1$ yrs; Body Fat: $13.1 \pm 1.5 \%$ ) were randomly assigned to ingest either $330 \mathrm{mg} /$ day of 3b-hydroxy-5aandrost-1-en-17-one (PROHORMONE; $\mathrm{n}=9$ ) or $330 \mathrm{mg} /$ day of sugar (PLACEBO; $\mathrm{n}=8$ ) and complete a 4 week (16 session) structured resistance-training program. The total testosterone (TT), free testosterone (FT), sex hormone binding globulin (SHBG), and free androgen index (FAI) were assessed at onset and termination of the study. Results: The PROHORMONE group decreased in TT (28\%) and SHBG (68\%) and increased in FAI (157\%). These values were virtually unchanged in the PLACEBO group TT $(+7 \%)$, SHBG $(+1 \%)$, and FAI $(+10 \%)$. FT did not change from PRE to POST in either PROHORMONE $(3 \%)$ or PLACEBO $(4 \%)$. The interaction between condition (PROHORMONE or PLACEBO) and time point (PRE or POST supplementation) was assessed via a 2-Factor Repeated-Measures ANOVA, and was significant for each variable (all $\mathrm{p}<0.01)$. Tukey post-hocs were used where appropriate. Conclusion: Our present data suggest that the anabolic effect of 3b-hydroxy-5a-androst-1-en-17-one is mediated via downregulation of SHBG. The resulting elevation in FAI contributes to greater peripheral androgen bioavailability that may improve body composition and muscular strength. 